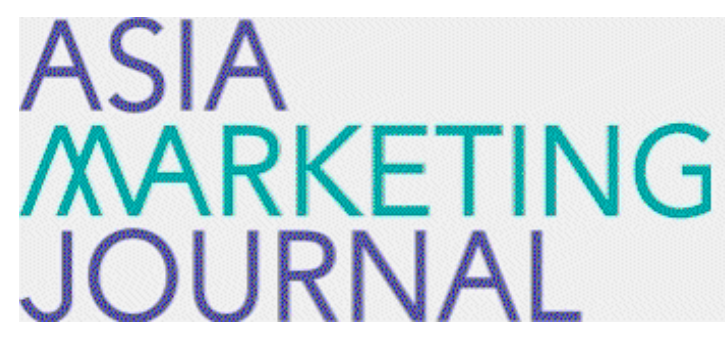

ASIA MARKETING JOURNAL

Volume 15 | Issue 3

Article 9

10-30-2013

\title{
Applying the Multiple Cue Probability Learning
}

So Won Ahn

Ju Young Kim

Young Won $\mathrm{Ha}$

Follow this and additional works at: https://amj.kma.re.kr/journal

Part of the Marketing Commons

\section{Recommended Citation}

Ahn, So Won; Kim, Ju Young; and Ha, Young Won (2013) "Applying the Multiple Cue Probability Learning," Asia Marketing Journal: Vol. 15 : Iss. 3 , Article 9.

Available at: https://doi.org/10.53728/2765-6500.1563

This Article is brought to you for free and open access by Asia Marketing Journal. It has been accepted for inclusion in Asia Marketing Journal by an authorized editor of Asia Marketing Journal. 


\title{
Applying the Multiple Cue Probability Learning to Consumer Learning*
}

\author{
Sowon Ahn** \\ Juyoung Kim*** \\ Young-Won $\mathrm{Ha}^{*}$
}

In the present study, we apply the multiple cue probability learning (MCPL) paradigm to examine consumer learning from feedback in repeated trials. This paradigm is useful in investigating consumer learning, especially learning the relationships between the overall quality and attributes. With this paradigm, we can analyze what people learn from repeated trials by using the lens model, i.e., whether it is knowledge or consistency. In addition to introducing this paradigm, we aim to demonstrate that knowledge people gain from repeated trials with feedback is robust enough to weaken one of the most often examined contextual effects, the asymmetric dominance effect.

The experiment consists of learning session and a choice task and stimuli are sport rafting boats with motor engines. During the learning session, the participants are shown an option with three attributes and are asked to evaluate its overall quality and type in a number between 0 and 100 . Then an expert's evaluation, a number between 0 and 100, is provided as feedback. This trial is repeated fifteen times with different sets of attributes, which comprises one learning session. Depending on the conditions, the participants do one (low) or three (high) learning sessions or do not go through any learning session (no learning). After learning session, the participants then are provided with either a core or an extended choice set to make a choice to examine if learning from feedback would weaken the asymmetric dominance effect. The experiment uses a between-subjects experimental design ( $2 \times 3$ : core set vs. extended set: no vs. low vs. high learning).

The results show that the participants evaluate the overall qualities more accurately with learning. They learn the true trade-off rule between attributes (increase in knowledge) and become more consistent in their evaluations. Regarding the choice task, there is a significant decrease in the percentage of choosing the target option in the extended sets with learning, which clearly demonstrates that

* This study was supported by the Sogang University Research Fund (201110066.01)

** Assistant Professor. Department of Business Administration, Seoul National University of Science and Technology (sahn@seoultech.ac.kr)

*** Professor, School of Business, Sogang University(jkimsg@sogang.ac.kr). Corresponding author

**** Professor, School of Business, Sogang University(ywha@sogang.ac.kr) 
learning decreases the magnitude of the asymmetric dominance effect. However, these results are significant only when no learning condition is compared either to low or high learning condition. There is no significant result between low and high learning conditions, which may be due to fatigue or reflect the characteristics of learning curve.

The present study introduces the MCPL paradigm in examining consumer learning and demonstrates that learning from feedback increases both knowledge and consistency and weakens the asymmetric dominance effect. The latter result may suggest that the previous demonstrations of the asymmetric dominance effect are somewhat exaggerated. In a single choice setting, people do not have enough information or experience about the stimuli, which may lead them to depend mostly on the contextual structure among options.

In the future, more realistic stimuli and real experts' judgments can be used to increase the external validity of study results. In addition, consumers often learn through repeated choices in real consumer settings. Therefore, what consumers learn from feedback in repeated choices would be an interesting topic to investigate.

Key words: Learning, Feedback, Multiple Cue Probability Learning, Lens Model, Knowledge, Consistency, Asymmetric Dominance Effect

\section{Introduction}

Susan decides to buy a mountain bike for outdoor activities. She visits Amazon and types 'mountain bike', and there are almost seventy thousand bikes. Overwhelmed by too much information, she notice star ratings made by other customers and experts. After looking through different options and reading a bike-buying guide, she chooses three or four attributes to consider. Then, she makes an evaluation for a product and sees a star rating that other customers have made. After observing a poor rating of the product, she moves to another product. She repeats this process several times and makes a final decision.

This short story illustrates the kind of situation to be investigated by the present study: learning from outcome feedback (i.e., overall quality evaluation of products, hereafter just 'feedback'). Consumer learning from feedback has been thought of as somewhat unrealistic and infrequent in the real world. These days, however, the online shopping environment provides consumers with a huge amount of information, some of which can act as feedback. For example, many shopping sites provide customer reviews such as star ratings.

In academics, most researchers admit that consumer learning is an important topic in consumer research, but direct investigation of 
this topic has been very limited (Huffman and Houston 1993: Hutchinson and Alba 1991). It is often believed that consumers acquire knowledge on the relationships that exist among and between products and attributes, but there are little studies examining how this knowledge is acquired. A few studies have examined learning and they have been conducted in the context of classical conditioning, covariation assessment, concept formation and categorization, hypothesis testing, and analogy (Allen and Janiszewski 1989; Bettman, John and Scott 1986: Gregan-Paxton and John 1997: Hoch and Ha 1986: Hutchinson and Alba 1991: Van Osselaer and Alba 2001).

The present study aims to introduce a useful paradigm in investigating consumer learning, especially learning the relationships between the overall quality and attributes. This paradigm is from psychology and called the multiple cue probability learning (MCPL), which observes learning over repeated trials with feedback (Karelaia and Hogarth 2008). With this paradigm, we can examine what people learn from repeated trials, i.e., whether it is knowledge or consistency. In addition to introducing the MCPL paradigm to consumer learning, we aim to demonstrate that knowledge people gain from repeated trials with feedback is robust enough to weaken one of the most often examined contextual effects, the asymmetric dominance effect.

\section{Theoretical Background}

\subsection{Multiple Cue Probability Learning}

In MCPL, the learning processes are conceptualized with three basic elements: a criterion $\left(\mathrm{Y}_{\mathrm{e}}\right)$, a set of cues $\left(\mathrm{x}_{1}, \mathrm{x}_{2}, \cdots, \mathrm{x}_{\mathrm{n}}\right)$ and feedback (Klayman 1988). In a traditional MCPL study, the task is often an evaluation or estimation $\left(\mathrm{Y}_{\mathrm{s}}\right)$. For example, you receive attribute information of products and are asked to evaluate the overall quality. Here, attribute information is a set of cues that are meant to be used to evaluate the overall quality, which is the criterion value. A researcher generally has set up a rule in advance that characterizes the relationship between the cues and the criterion and produces criterion values according to the rule, which is unknown to people and which they are supposed to figure out. The rule has random errors in it to make the relationship probabilistic.

In MCPL, learning performance is analyzed with lens model. The lens model is a statistical method to capture human judgment or learning processes (Brunswik 1952: Hammond et al. 1964: Hursch et al. 1964). The criterion values $\left(\mathrm{Y}_{\mathrm{e}}\right)$ are produced by the rule set by $\mathrm{re}^{-}$ searchers (Eq. 1) and evaluations $\left(Y_{\mathrm{s}}\right)$ are modeled by using a multiple regression with the cues as predictors $\left(\mathrm{x}_{1}, \mathrm{x}_{2}, \cdots, \mathrm{x}_{\mathrm{n}}\right)$ (Eq. 2). In Equation 1 and $2, Z_{e}$ and $Z_{s}$, refer to ran- 
dom error in each model.

$$
\begin{aligned}
& Y_{e}=\hat{Y}_{e}+Z_{e} \quad\left(\hat{Y}_{e}=\sum \beta_{i e} X_{i}\right) \\
& Y_{s}=\hat{Y}_{s}+Z_{s} \quad\left(\hat{Y}_{s}=\sum \beta_{i s} X_{i}\right) \\
& r_{a}=G_{s} R_{e}+C \sqrt{ }\left(1-R_{s}^{2}\right) \sqrt{ }\left(1-R_{e}^{2}\right)
\end{aligned}
$$

To see how well each participant performs in the lens model analysis, his or her evaluations and the criterion values are correlated. This correlation, which is $r_{a}$ in the lens model equation (Eq. 3), gives the overall accuracy or "achievement" of a participant's performance, i.e., how well evaluations correspond to the criterion values (Tucker 1964). Then, the overall achievement is decomposed into several components as follows. "Knowledge" (G) is defined as the correlation between the fitted values of evaluations, $\hat{Y}_{\mathrm{s}}$, and the fitted values of the criterion, $\hat{\mathrm{Y}}_{\mathrm{e}}$, and "measures the extent to which the participant has correctly detected properties of the task (p. 60)" (Hammond and Summers 1965). For example, a high G indicates that the systematic part of participants' evaluations is isomorphic with that of the criterion, and this implies that participants have correctly figured out which cues are relevant and how those cues combine to determine overall quality and used that knowledge to make evaluations. "Consistency" or predictability of a participant's evaluations $\left(R_{s}\right)$ is given by the correlation between a participant's evaluations, $Y_{s}$, and fitted values of evaluations, $\hat{Y}_{s} . R_{s}$ and $G$ are statistically in- dependent, so a person may have a high G, but a rather low $R_{s}$, which indicates that the person has not applied the knowledge he or she acquired consistently (Deane et al. 1972). "Consistency" or predictability of the criterion $\left(R_{e}\right)$ is the correlation between the criterion, $\mathrm{Y}_{\mathrm{e}}$, and fitted values of the criterion, $\hat{\mathrm{Y}}_{\mathrm{e}} \mathrm{C}$ is the correlation between the residuals, $Z_{e}$ and

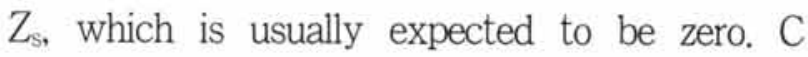
captures the part of achievement related to cues that have not been included in the model, nonlinearities in the cue-criterion relations, and possible configurality (interactions between cues).

This decomposition of overall achievement into different indices is helpful because it informs us which indices, which are psychologically distinctive, contribute to an increase in $r_{a}$ (Hammond and Summers 1965). An increase in $r_{a}$ can be obtained either by an increase in task knowledge $(\mathrm{G})$ or by an increase in consistency of evaluations $\left(R_{s}\right)$, or both.

In MCPL studies, there are two types of feedback, outcome and cognitive feedback. Outcome feedback provides people with the true value of the criterion immediately after the evaluation. Cognitive feedback provides either task information (the relationships between cues and the criterion), cognitive information (the relationships between cues and a person's evaluations), or functional validity information (the relationships between the criterion and a person's evaluations). Cognitive feedback is often delivered after multiple trials 
and is more efficient than the outcome feedback in helping people infer the underlying rule (e.g., Hammond et al. 1973).

In the current study, the participants are provided with outcome feedback based on a rule set up by researchers because this type of feedback is more available to consumers in real life than cognitive feedback. As in MCPL studies, the participants of the present study are exposed to various combinations of attribute values one by one and asked to evaluate the overall quality of a product and receive feedback immediately after each evaluation. By receiving feedback over repeated trials, the participants are expected to learn how to evaluate overall qualities of products.

\subsection{Knowledge and the Asymmetric Dominance Effect}

There are two alternatives, which are similar in overall attractiveness and none dominates the other. Now, add an alternative that is asymmetrically dominated by one of the existing alternatives. Then, due to the entrance of this new alternative, one of the existing alternatives, which dominates the new alternative. is chosen more often than when there are only two. This phenomenon is called the asymmetric dominance effect' and first introduced by Huber, Payne and Puto (1982). Since its introduction, the effect has been one of the main topics in decision research and how knowledge affects the effect was examined as well. Researchers predicted that more knowledge would weaken the asymmetric dominance effect because the effect is contextual and it would have less influence on the people's choice if they knew more about the relationships between attributes and overall quality. The results from related studies, however, have shown that the relationship was not so simple.

Although Ratneshwar, Shocker and Stewart (1987) reported that more knowledge indeed weakened the asymmetric dominance effect for the product class that participants were most knowledgeable in, Ha and Chae (1993) proposed a more complex relationship between familiarity and the asymmetric dominance effect. They demonstrated that the highest level of asymmetric dominance effect emerged among participants with moderate familiarity, in an inverted $\mathrm{U}$ relationship between familiarity and the magnitude of the asymmetric dominance effect. Their explanation is that people with low familiarity do not have enough ability to process product information, whereas people with high familiarity do not have enough motivation to process the information. On the other hand, people with moderate familiarity have a certain level of processing ability and motivation, and thus utilize the given information to a full degree. As a result of processing, they become more susceptible to a contextual suggestion and show a higher degree of the asymmetric dominance effect than people with low 
or high familiarity.

Sen (1998) attempted to explain these rather complex relationships by suggesting that the information mode (numerical vs. verbal) moderated the relationships between knowledge and the asymmetric dominance effect. When the attribute information was presented numerically, knowledge decreased the effect, but increased the effect when presented verbally. The rationale is as follows. Experts are more sensitive to the differences in attribute levels. And this sensitivity is increased when attribute information is presented verbally. Therefore, experts are more vulnerable to the asymmetric dominance effect when attribute information is presented verbally. However, experts demonstrate less asymmetric dominance effect when attribute information is presented numerically because they are better at making trade-offs and this superior ability becomes more apparent when attribute information is presented numerically.

In the present research, all attribute information is presented numerically because we want to demonstrate the impact of learning from feedback on the asymmetric dominance effect. People are expected to become more knowledgeable about how to evaluate the overall qualities with attributes as the learning from feedback proceeds. In turn, the magnitude of the asymmetric dominance effect is expected to decrease. Previous research on how knowledge affects the asymmetric dominance effect has compared people with different degrees of knowledge, but the current study aims to examine how knowledge formed over time within-a-person affects the asymmetric dominance effect.

It is expected that learning from repeated trials with feedback would increase both knowledge and consistency (Hypothesis 1) and knowledge would weaken the attraction effect (Hypothesis 2). Here are hypotheses to be tested in this study.

Hypothesis 1-A: As people repeat evaluations with feedback, they become more knowledgeable about the trade-off rule (increase in G).

Hypothesis 1-B: As people repeat evaluations with feedback, they make evaluations more consistently (increase in $R_{s}$ ).

Hypothesis 2-A: As people repeat evaluations with feedback, they choose the best option more often in a choice task.

Hypothesis 2-B: As people repeat evaluations with feedback, the magnitude of the asymmetric dominance effect decreases in a choice task.

\section{Experiment}

Multiple cue information, which involves the attribute values in this case, is given to the participants and they are asked to evaluate the 
overall quality numerically using numbers between 0 and 100 based on the given attribute values. Feedback is provided immediately after the evaluation. The feedback is introduced as an expert's evaluation that is generated based on a trade-off rule predetermined by the researchers. This process is repeated with various combinations of attribute values.

\subsection{Method}

The participants are 272 college students in business schools in Seoul, Korea (males: 144, females: 128). All participants volunteer to join the study and are rewarded with small gifts, such as ball-point pens, snacks and coffee coupons.

As stimuli, sport rafting boats with motor engines are selected because the participants may have heard about them but are not likely to have had a chance to buy or evaluate, which indicates room for learning. In addition, overall quality of sport rafting boats can be determined objectively based on attributes. Each rafting boat is described with three attributes. The three attributes selected are the diameter of the rubber tube $(30 \sim 60$ inch), the maximum power of the motor engine ( $5 \sim 80 \mathrm{HP})$, and the degree of shape distortion (40 200 HR). The first two attributes are positively correlated with the overall quality, whereas the third attribute is negatively correlated with the overall quality. To make an option, the three attribute levels of each option are chosen ran- domly within the prescribed ranges and the relative weights of the three attributes are set to 35 (diameter of a boat) vs. 20 (degree of shape distortion) and 45 (power of engine) to determine overall quality. And to make the relationships probabilistic, 5\% random error is added to the true quality based on the tradeoff rule.

The experiment consists of learning session and a choice. During the learning session, the participants are shown an option with three attributes and are asked to evaluate its overall quality and type in a number between 0 and 100. Then an expert's evaluation, a number between 0 and 100, is provided as feedback. This trial is repeated fifteen times with different sets of attributes, which comprises one learning session. Depending on the conditions. the participants do one or three learning sessions or do not go through any learning session. After the learning session, they are provided with a choice set and are asked to choose the best quality option from it. The choice set consists of three or four options depending on the conditions. In the core set, there are three options, none of which dominates the others. To make a corresponding extended set, one of the options is chosen randomly as a target and a decoy is made by lowering $2 \sim 3 \%$ of the values of all three attributes from the target. It is then added to the set (Table 1).

The experiment uses a between-subjects experimental design $(2 \times 3$ : core set vs. ex- 
〈Table 1〉 A choice set

\begin{tabular}{cccc}
\hline & $\begin{array}{r}\text { Diameter of rubber tube } \\
(30 \sim 60 \text { inch })\end{array}$ & $\begin{array}{c}\text { Degree of shape distortion } \\
(40 \sim 200 \mathrm{HR})\end{array}$ & $\begin{array}{c}\text { Maximum power of motor engine } \\
(5 \sim 80 \mathrm{HP})\end{array}$ \\
\hline Target & 45.3 & 107 & 68.8 \\
Worst & 48.9 & 72 & 35.0 \\
Best & 44.7 & 96 & 72.6 \\
Decoy & 45.0 & 112 & 65.4 \\
\hline
\end{tabular}

tended set: no vs. low vs. high learning). The choice set and the degree of learning are manipulated. The 272 participants are divided into six groups: 60 in the core set no-learning condition, 64 in the extended set no-learning condition, 38 in the core set under the lowlearning condition, 32 in the core set under the high-learning condition, 41 in the extended set under the low-learning condition, and 37 in the extended set under the high-learning condition. The no-learning conditions are used to determine if the asymmetric dominance effect is present as a baseline and the participants in those conditions merely choose an option from the choice set without any learning session.

The experiment is run on computer individually and started with a brief introduction to the task.

\subsection{Results}

$\mathrm{H1}$ inquires about whether people evaluate the overall qualities more accurately with more learning. Table 2 lists the means of $r_{a}, G, R_{S}$ and $\mathrm{C}$ for a session of the low-learning con- dition and three sessions of the high-learning condition. Repeated measurement ANOVA is carried out for the high-learning condition, the learning session as a within-subject factor and the type of choice set as a between-subjects factor. As expected, no main effect of the type of choice set is observed, but the main effects of learning session are observed (Table 2). When the session means of the high-learning condition are compared, the session pairs 1 and 2 and 1 and 3 are significant $(p<.05)$. Although the session pair 2 and 3 is not significant, the participants exhibit an increase in all the indices, as shown in Table 2. The results suggest that the participants learn the true trade-off rule between attributes (as appeared in the increases in G: H1-A) and become more consistent in their evaluations (as appeared in increases in $\mathrm{R}_{\mathrm{s}}$ : $\left.\mathrm{H} 1-\mathrm{B}\right)$, which results in increases in the overall achievement of $r_{a}$. The Cs of sessions 1 and 2 are approximately zero as expected, but the $\mathrm{C}$ of session 3 is significantly different from zero $(t(68)=-3.85, p$ $<$.001) and significantly lower than the Cs of sessions 1 and $2(p<.05)$. This increase in the 
〈Table 2〉 The means of lens model indices $(S D)$

\begin{tabular}{cccccc}
\hline & Low learning & \multicolumn{5}{c}{ High learning $(\mathrm{n}=69)$} \\
& $(\mathrm{n}=79)$ & Session 1 & Session 2 & Session 3 & $F(2,134)$ \\
\hline ra & $0.29(.31)$ & $0.30(.37)$ & $0.56(.36)$ & $0.60(.33)$ & $39.24^{* * *}$ \\
G & $0.40(.50)$ & $0.43(.55)$ & $0.66(.45)$ & $0.71(.40)$ & $14.22^{* * *}$ \\
Rs & $0.61(.22)$ & $0.64(.19)$ & $0.77(.21)$ & $0.80(.15)$ & $31.06^{* * *}$ \\
C & $-0.03(.28)$ & $0.01(.31)$ & $0.00(.24)$ & $-0.14(.31)$ & $6.52^{* *}$ \\
\hline
\end{tabular}

${ }^{* *} p<.01,{ }^{* * *} p<.001$

absolute value of $\mathrm{C}$ in session 3 may indicate the participants' boredom or fatigue or their attempt to try non-linear or configural rules as they gain some knowledge about the products.

$\mathrm{H} 2$ inquires about choice. A part of $\mathrm{H} 2$ is tested by comparing the percentage of participants choosing the best option under the three different learning conditions (Figure 1). In the core set, the percentage of participants choosing the best option with no learning (52.4\%) increases significantly to $71.1 \%$ under the low-learning condition $(z=1.75, p<0.05)$ and $78.1 \%$ under the high-learning condition $(z=2.42, p<0.01)$. For the extended set, the percentage of participants choosing the best option with no learning (31.6\%) increases significantly to $63.4 \%$ under the low-learning condition $(z=2.99, p<0.01)$ and $78.4 \%$ under the high-learning condition $(z=4.62, p<$ $0.01)$. The percentages of choosing the best option increase in both sets and thus $\mathrm{H} 2-\mathrm{A}$ is confirmed.

$\mathrm{H} 2-\mathrm{B}$ is tested by comparing the magnitudes of the asymmetric dominance effects under

〈Figure 1> Percentage of the best choice under different conditions

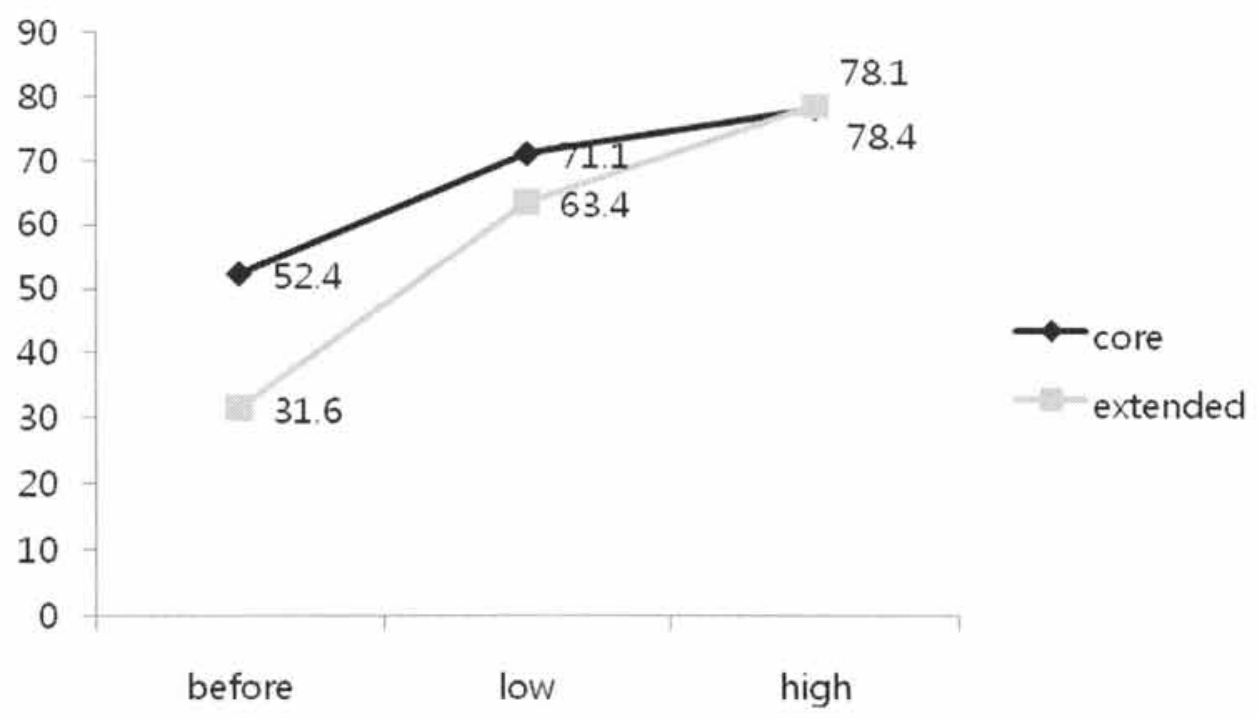


different learning conditions (Figure 2). We compare the percentage that chooses a target in a core set with that in an extended set. The magnitude of the asymmetric dominance effect decreases from $15.0 \%$ to $4.3 \%$ when comparing the no-learning condition with the low-learning condition, from $4.3 \%$ to $-5.3 \%$ when comparing the low-learning condition to the high-learning condition, and from $15.0 \%$ to $-5.3 \%$ when comparing the no-learning condition to the high-learning condition, but none of the differences is significant $(p>05)$. Another way to examine the asymmetric dominance effect is to check whether the percentage of participants choosing the target option decreases as learning proceeds (Simonson and Sela 2011). The percentage of participants choosing the target option in the extended set shows a significant decrease from $57.9 \%$ (no-learning condition) to $12.2 \%$ (low-learning condition) $(z=4.81, p<$
$.001)$, and the percentage slightly increases to 13.5\% under the high-learning condition. From no-learning condition to low-learning condition, the percentage of choosing the target option that is helped by the existence of decoy decreases. However, it does not decrease at the high-learning condition.

Although the changes from the low- to the high-learning conditions do not always decrease and the differences are not significant, the significant changes from the no to the low-learning conditions clearly demonstrate that the number of choices of the target option decreases with learning. The statistical test partially confirms $\mathrm{H} 2-\mathrm{B}$, but the data clearly follows the direction of $\mathrm{H} 2-\mathrm{B}$. The small change between the low- and the high-learning conditions may have been due to fatigue or reflect the characteristics of learning curve. Usually there is a noticeable improvement in the early

〈Figure 2〉 Percentage of the target option under different conditions

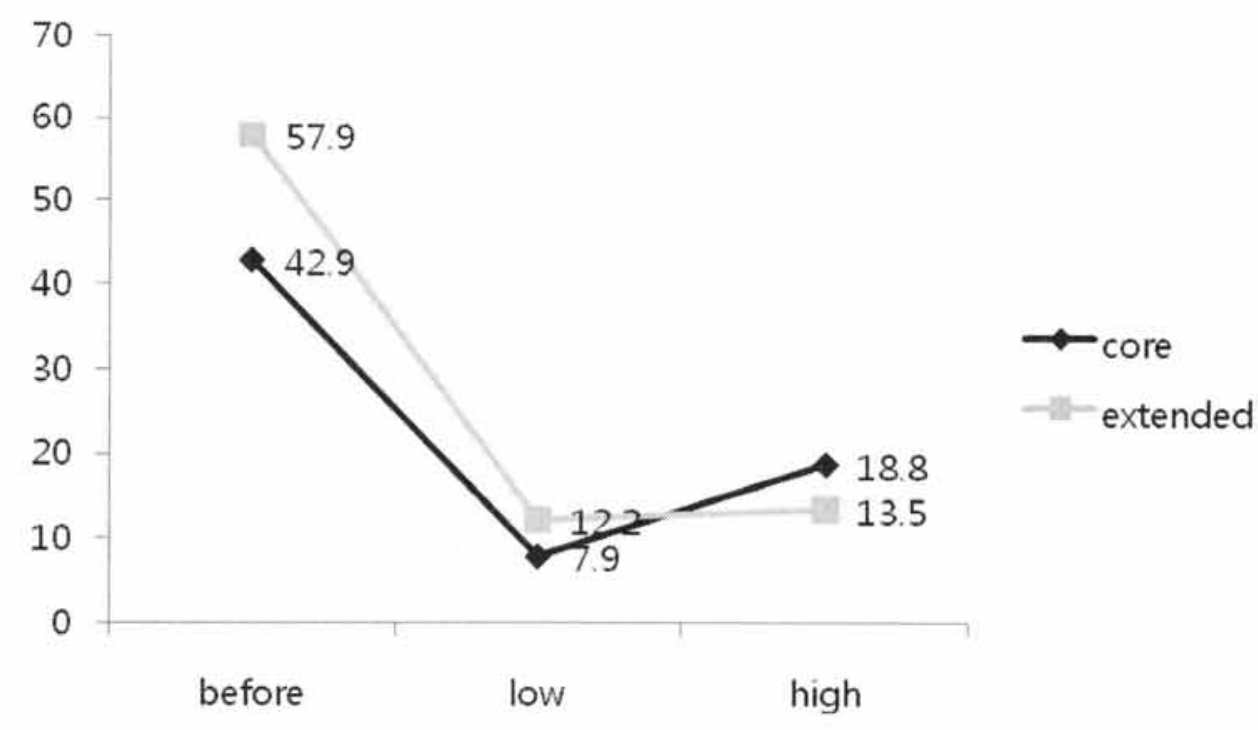

168 ASIA MARKETING JOURNAL Vol. 15 NNo. 03 October 2013 
stage of learning, but the speed and the amount of learning decrease as learning goes on (Bills 1934).

\section{General Discussion}

The current study introduces the MCPL paradigm in examining consumer learning and demonstrates that learning from feedback weakens the asymmetric dominance effect. The MCPL paradigm is useful in that it shows what consumers learn from feedback in repeated trials. Based on correlational statistics, the lens model provides us with the neat indices of performance, i.e., knowledge and consistency. In addition, it introduces various task conditions that can affect learning, such as the number of cues, types of cues (given vs. achieved), cue redundancy (none vs. some vs. high), function form of the criterion (linear vs. nonlinear), cue weights in the criterion (compensatory vs. noncompensatory vs. equal weighting), context (abstract vs. concrete), type of study (laboratory vs. field), and so on (Kaleraia and Hogarth 2008).

The current study uses hypothetical cues, cue values, and the criterion values, but to increase the external validity of study results, more realistic stimuli and real experts' judgments can be used instead. In real consumer settings, there can be more than three attrib- utes and consumers have to figure out which attributes are valid in predicting the overall quality instead of given attributes. The true rule constructed in the current study is linear and compensatory, but there can be product categories where different rules apply. Varying these task characteristics may result in different degrees of learning and how these affect learning in consumer settings may give rise to a series of research. Recently, there have been studies demonstrating how the lens model research can be conducted in more representative and natural environments (e.g., Gosling, Ko, Mannarelli, and Morris 2002: Vazire and Gosling 2004), and these attempts can be applied to consumer settings to broaden the understanding of consumer learning.

Prior research has shown that knowledge moderates the asymmetric dominance effect (though the relationship appears rather complex) and has compared groups with different degrees of knowledge. The present study also examines the effect of knowledge on the asymmetric dominance effect but differs from previous research in that it examined the effect within subjects, not between subjects, and thus directly addresses the issue of learning regarding the asymmetric dominance effect. In addition, the result that learning from feedback weakens the effect may suggest that the previous demonstrations of the asymmetric dominance effect are somewhat exaggerated. In a single choice setting, people do not have enough 
information or experience about the stimuli, which may lead them to depend mostly on the contextual structure among options.

In the current study, learning from feedback occurs through repeated evaluations of qualities. We use the evaluation task to apply the lens model analysis directly to our task. In real consumer settings, however, consumers often learn through repeated choices. Moreover, there have been studies demonstrating that in repeated choices people behave differently from a single choice situation (Benartzi and Thaler 1999; Keren 1991). Therefore, what consumers learn from feedback in repeated choices would be an interesting topic to investigate in the future. For example, study on choice among hedonic, utilitarian, and mixed attribute products would be enriched if changes in repeated choices with adding different attributes are investigated (Yi and Muhn 2013).

〈Received July 27. 2013〉

〈1st Revised September 15. 2013〉 〈2nd Revised September 30. 2013〉

〈Accepted October 8. 2013〉

\section{References}

Allen, Chris T. and Chris A. Janiszewski (1989), "Assessing the Role of Contingency Awareness in Attitudinal Conditioning with Implications for Advertising Research,"
Journal of Marketing Research, 26 (1), $30-43$.

Benartzi, Shlomo and Richard H. Thaler (1999), "Risk Aversion or Myopia? Choices in $\mathrm{Re}^{-}$ peated Gambles and Retirement Investments," Management Science, 45 (3), 36481.

Bettman, James R., Deborah R. John and Carol A. Scott (1986), "Covariation Assessment by Consumers," Journal of Consumer Research, 13 (3), 316-26.

Bills, Authur Gilbert (1934), General Experimental Psychology. Longmans Psychology Series. (pp. 192-215). New York, NY: Longmans, Green and Co.

Brunswik, Egon (1952), The Conceptual Framework of Psychology. Chicago: University of Chicago Press.

Deane, Donald H., Kenneth R. Hammond and David A. Summers (1972), “Acquisition and Application of Knowledge in Complex Inference Tasks," Journal of Experimental Psychology, 92 (1), 20-6.

Gosling, Samuel D., Sei Jin Ko, Thomas Mannarelli and Margaret E. Morris (2002), "A Room with a Cue: Personality Judgments Based on Offices and Bedrooms," Journal of Personality and Social Psychology, 82 (3), 379-98.

Gregan-Paxton, Jennifer and Deborah Roedder John (1997), "Consumer learning by analogy: A model of internal knowledge transfer," Journal of Consumer Research, 24 
(3), 266-84.

Ha, Young-Won and Jung-Ho Chae (1993), "The Asymmetric Dominance Effect: The Influence of Stimulus Range and Frequency," Korean Management Review. 23 (November), 201-32.

Hammond, Kenneth R., Carolyn J. Hursch and Frederick J. Todd (1964), “Analyzing the Components of Clinical Inference," Psychological Review, 71 (6), 438-56.

Hammond, Kenneth R. and David A. Summers (1965), “Cognitive Dependence on Linear and Non-linear Cues," Psychological Review. 72 (3), 215 - 24.

Hammond, Kenneth R., David A. Summers and Donald H. Deane (1973), "Negative Effects of Outcome Feedback in MultipleCue Probability Learning," Organizational Behavior and Human Performance, 9 (February), 30-4.

Hoch, Stephen J. and Young-Won Ha (1986), "Consumer Learning: Advertising and the Ambiguity of Product Experience," Journal of Consumer Research, 13 (2), 221-33.

Huber, Joel, John W. Payne, and Christopher Puto (1982), “Adding Asymmetrically Dominated Alternatives: Violations of $\mathrm{Re}^{-}$ gularity and the Similarity Hypothesis," Journal of Consumer Research, 9 (June), 90-98.

Huffman, Cynthia and Michael J. Houston (1993), “Goal-Oriented Experiences and the Development of Knowledge," Journal of Consumer Research, 20 (September), 190-207.

Hursch, Carolyn J., Kenneth R. Hammond and Jack L. Hursch (1964), “Some Methodological Considerations in Multiple-Probability Studies," Psychological Review, 71 (1), 42-60.

Hutchinson, J. Wesley and Joseph W. Alba (1991), "Ignoring Irrelevant Information: Situational Determinants of Consumer Learning," Journal of Consumer Research, 18 (3), 325-45.

Karelaia, Natalia and Robin M. Hogarth (2008), "Determinants of Linear Judgment: A Meta-Analysis of Lens Model Studies," Psychological Bulletin, 134 (3), 404-26.

Keren, Gideon (1991), “Additional Tests of Utility Theory under Unique and Repeated Conditions," Journal of Behavioral Decision Making, 4 (4), 297-304.

Klayman, Joshua (1988), “On the How and Why (not) of Learning from Outcomes," In Human Judgment: The SJT View. Brehmer, Berndt and C. R. B. Joyce, eds. Elsevier Science Publishers.

Ratneshwar, Srinivasan, Allan D. Shocker, and David W. Stewart (1987), “Toward Understanding the Asymmetric Dominance Effect: The Implications of Product Stimulus Meaningfulness and Familiarity," Journal of Consumer Research, 13 (March), 520-33.

Sen, Sankar (1998), "Knowledge, Information 
Mode, and the Asymmetric Dominance Effect," Journal of Consumer Research, 25 (June), 64-77.

Simonson, Itamar, and Aner Sela (2011), "On the heritability of consumer decision making: An exploratory approach for studying genetic effects on judgment and choice," Journal of Consumer Research, 37 (6), 951-66.

Tucker, Ledyard R. (1964), "A Suggested Alternative Formulation in the Developments by Hursch, Hammond, and Hursch and by Hammond, Hursch, and Todd," Psychological Review, 71 (6), 528-30.
Van Osselaer, Stijn MJ and Joseph W. Alba (2000). "Consumer learning and brand equity," Journal of consumer research, 27 (1), 1-16.

Vazire, Simine and Samuel D. Gosling (2004), "E-perceptions: Personality Impressions Based on Personal Websites," Journal of Personality and Social Psychology, 87 (1), 123-32.

Yi, Youjae and Sunhee Muhn (2013), "Mixed Products: How Adding Different Attributes Influences Consumer Perceptions and Product Evaluation," Asia Marketing Journal, 15(1), 83-105 